\title{
Meta-Analysis of the Clinical Value of Danshen Injection and Huangqi Injection in Liver Cirrhosis
}

\author{
Changtai Zhu, ${ }^{1,2}$ Hao Cao, ${ }^{3}$ Xifa Zhou, ${ }^{1}$ Chunlei Dong, ${ }^{2}$ Judong Luo, ${ }^{1}$ Changsong Zhang, \\ Jinming Liu, ${ }^{5}$ and Yang Ling ${ }^{1,4}$ \\ ${ }^{1}$ Department of Radiation Oncology, Changzhou Tumor Hospital Soochow University, Changzhou 213000, China \\ ${ }^{2}$ Department of Laboratory Medicine, Changzhou Tumor Hospital Soochow University, Changzhou 213000, China \\ ${ }^{3}$ Department of Cardiothoracic Surgery, Shanghai East Hospital, Tongji University School of Medicine, Shanghai, China \\ ${ }^{4}$ Department of Oncology, Changzhou Tumor Hospital Soochow University, Changzhou 213000, China \\ ${ }^{5}$ Department of Respiratory Medicine, Shanghai Pulmonary Hospital, Tongji University School of Medicine, Shanghai, China
}

Correspondence should be addressed to Jinming Liu; jinming2007@163.com and Yang Ling; lingyang11566@163.com

Received 20 May 2013; Accepted 24 July 2013

Academic Editor: Qing He

Copyright (c) 2013 Changtai Zhu et al. This is an open access article distributed under the Creative Commons Attribution License, which permits unrestricted use, distribution, and reproduction in any medium, provided the original work is properly cited.

\begin{abstract}
Objective. To evaluate the clinical value of Danshen injection and Huangqi injection for the treatment of liver cirrhosis. Methods. The Chinese Biomedical Literature Database (CBM), Chinese Scientific Journals Full-Text Database (VIP), Wanfang Database, China National Knowledge Infrastructure (CNKI), PubMed, and EMBASE database were searched to collect the literatures about the randomized controlled trials involving the treatment of liver cirrhosis with Danshen injection combined with Huangqi injection, and the data analyses were performed using RevMan 4.2 software. Results. A total of 11 studies involving 1086 patients (trials group: 554 cases, control group: 532 cases) were included in this study. Compared with those in control group, the meta-analysis showedthat the total effectiveness rate and the level of serum albumin increased, while serum total bilirubin, alanine transmninase, type III procollagen, hyaluronic acid, laminin, and type-IV collagen decreased in trials group. The Jadad score ranged from 1 to 2 and the funnel plot analysis suggests that publication bias may occur. Conclusions. Danshen injection combined with Huangqi injection may promote the curative efficacy of liver cirrhosis, which is a promising novel treatment approach. The exact outcome needs to perform rigorously designed, multicenter, and large randomized controlled trials.
\end{abstract}

\section{Introduction}

Liver fibrosis or cirrhosis is a common progressively pathological lesion of chronic liver diseases in response to various liver-damaging factors [1]. The main causes of liver fibrosis in industrialized countries include chronic HCV infection, alcohol abuse, and nonalcoholic steatohepatitis [2]. In China, cirrhosis is most commonly caused by hepatitis B and hepatitis $C$, because China has the largest number of hepatitis patients in the world. Of the 350 million to 400 million individuals worldwide infected with the hepatitis $\mathrm{B}$ virus (HBV), one-third reside in China, with 130 million carriers and 30 million chronically infected $[3,4]$. Presently, the treatment for liver cirrhosis remained difficult, due to the lack of effective approaches. In recent years, many clinical trials using Danshen injection and Huangqi injection for the treatment of liver cirrhosis have been reported in China, suggesting that the new therapeutic approach may have potential value. However, there lacked a systematical review about the issue. Therefore, we conducted a meta-analysis of RCTs to assess the clinical value of Danshen injection and Huangqi injection for the treatment of liver cirrhosis.

\section{Materials and Methods}

2.1. Inclusion Criteria. The studies were randomized controlled trials (RCTs) and quasi-randomized controlled trials. Clinical diagnosis must meet the diagnostic criteria for cirrhosis of the liver (Chinese Commission of Infectious and Parasitic Diseases, Viral Hepatitis Prevention and Treatment Programs). The trials group added Danshen or compound 


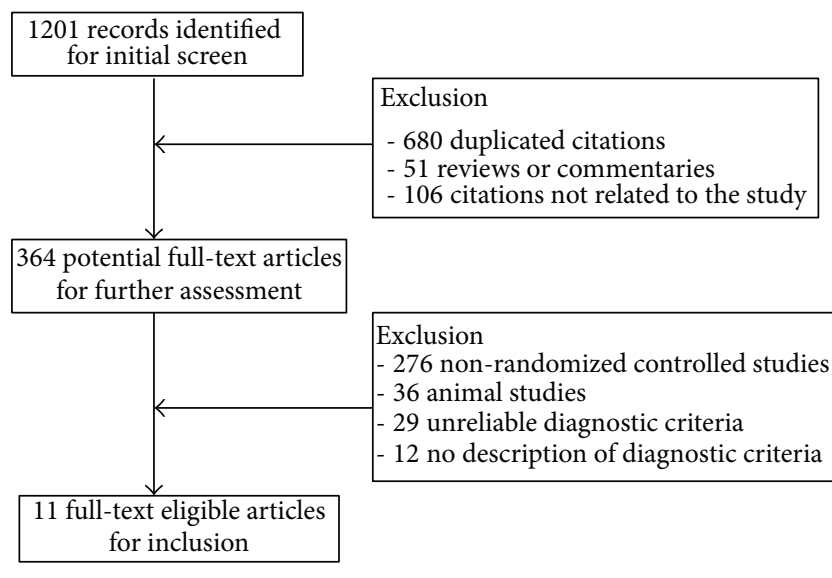

FIGURE 1: Flow diagram of the literature selection for this study.

Danshen injection and Huangqi injection apart from the drugs that were used by the control group. The outcome should include one or more indices as follows: (1) total efficacy rate; (2) serum indices of liver function: alanine aminotransferase (ALT), total bilirubin (TBIL), and albumin (ALB); (3) serum indices of liver fibrosis: hyaluronic acid (HA), type-IV collagen (VI-C), type-III procollagen (PC-III) and laminin (LN); (4) adverse events; E other data related to efficacy and outcome.

In this study, we had not set any restrictions on gender, race, and literature language.

2.2. Exclusion Criteria. Reviews, nonclinical studies, case observations, duplicated literatures, and noninjection formulae literatures were excluded.

2.3. Research Strategy and Data Extraction. Danshen, dan shen, huangqi, Beiqi, huang qi, salvia, salvia miltiorrhiza, astragalus, astragali, astragalus miltiorrhiza, Chinese traditional medicine herb, fibrosis, cirrhosis of liver, cirrhosis, liver cirrhosis, and liver fibrosis were selected as the search terms. The Chinese Biomedical Literature Database (CBM), China National Knowledge Infrastructure (CNKI), Chinese Scientific Journals Full-text Database (VIP), Wanfang Database, PubMed, and EMBASE database were searched by computer. Data extraction and quality assessment was independently performed by two researchers (C. Zhu and Y. Ling) and disagreements were resolved by consensus. The lack of information was supplemented by contact with the authors in charge of the clinical trials. Database retrieval process is shown in Figure 1. The methodological quality of trials was assessed by Jadad score. The randomization, concealment of allocation, blind methods, and loss of followup were considered as the scoring evidence.

2.4. Statistical Analysis. Statistical analysis was performed by Cochrane RevMan 4.2. Categorical variables were compared using relative risk (RR), and continuous variables were compared using weighted mean difference (WMD). Meanwhile, 95\% confidence interval (CI) was calculated. Chi-square test was used for the heterogeneity of inclusion trials. The heterogeneity data adopted the random effect model was used, otherwise fixed effect model. A funnel plot was used for assessing the potential publication bias.

\section{Results}

3.1. Characteristics of Included Studies. Eleven articles [515] involving 1086 subjects (trials group: 554 cases; control group: 532 cases) were included in this study. Male patients with liver cirrhosis were dominated in the subjects. The general characteristics of the study were shown in Table 1, and interventions, treatments and outcomes were seen in Table 2.

3.2. The Quality Assessment. The design features clarified as randomization, double-blinding, withdrawals/dropouts, and allocation concealment were shown in Table 3. The Jadad scores ranged from 1 to 2 . In this study, the curative effect assessment was performed by the clinicians and doubleblinding design was not adopted. But, the measurements of serum indices of liver function and liver fibrosis were independently conducted by laboratory staff, and the doubleblinding method was performed in all the included studies. However, the withdrawals/dropouts and allocation concealment were not conducted.

3.3. Meta-Analysis of Assessing the Total Effectiveness Rate. Four RCTs $[5,7,8,11]$ have evaluated the total efficacy rate. Heterogeneity analysis of the total efficacy rate was significant difference $(P<0.05)$, and a random effects model was used. The results showed the total efficacy rate in the trials group was higher than that in the control group $(\mathrm{RR}=1.22,95 \% \mathrm{CI}$ $(1.0,1.48) ; P<0.05)$ (Figure 2).

3.4. Meta-Analysis of the Serum Indices of Liver Function. The heterogeneity occurred in the data of ALT, TBIL, and ALB $(P<0.05)$; therefore, we adopted a random effects model. The results showed that the levels of ALT and TBIL in the trials group decreased significantly compared with the control group, while the ALB level increased. The WMDs with $95 \%$ CI of ALT, TBIL and ALB were -18.11 (-28.42, -7.81), -23.60 $(-35.39,-11.81)$ and $4.26(2.49,6.04)$, respectively. Forest plots were shown in Figure 3.

3.5. Meta-Analysis of the Serum Indices of Liver Fibrosis. Heterogeneity analyses implied that PC-III, HA, and VI-C fit random effects model, while LN should adopt a fixed effects model. Meta-analysis showed that the WMDs of PCIII, HA, LN, and VI-C with 95\% CI were -62.24 (-94.58, $-29.90),-144.60$ (-222.16, -67.04), -40.33 (-47.32, -33.33), and $-39.98(-52.17,-27.80)$, respectively, suggesting that the levels of serum PCIII, HA, LN, and VI-C in the trials group were lower than those in control group (Figures 4 and 5).

3.6. Liver Hemodynamics, $H B e A g$ Seroconversion and $H B V$ $D N A$. Wu et al. [12] reported hemodynamic changes in liver as follows: after 4 weeks of treatment, portal vein diameter, the inner diameter, and blood flow velocity of the splenic 
TABLE 1: Characteristics of the randomized controlled trials included in this study.

\begin{tabular}{|c|c|c|c|c|c|}
\hline Author (reference) & Published year & Cases T/C & $\begin{array}{c}\text { Age (years) } \\
\text { Range, mean }\end{array}$ & $\begin{array}{c}\text { Sex } \\
\text { Male/female }\end{array}$ & $\begin{array}{c}\text { History (years) } \\
\text { Range, mean }\end{array}$ \\
\hline Shen et al. [5] & 1999 & $39 / 35$ & $\begin{array}{l}\text { T: } 39-72,55.3 \\
\text { C: } 40-71,56.5\end{array}$ & $\begin{array}{l}\text { T: } 26 / 13 \\
\text { C: } 23 / 12\end{array}$ & $2-13,5.7$ \\
\hline Chen et al. [6] & 2000 & $80 / 40$ & NR & NR & NR \\
\hline Cai et al. [7] & 2001 & $44 / 40$ & $\begin{array}{c}\mathrm{T}: 8-51,29 \\
\mathrm{C}: 12-50,31\end{array}$ & $\begin{array}{l}\text { T: } 35 / 9 \\
\text { C: } 32 / 8\end{array}$ & $\begin{array}{l}\text { T: } 0.8-11,5.9 \\
\text { C: } 0.7-11,5.8\end{array}$ \\
\hline Zhou [8] & 2002 & $60 / 60$ & $\begin{array}{l}\text { T: } 28-70,49 \\
\text { C: } 26-68,47\end{array}$ & $\begin{array}{l}\mathrm{T}: 40 / 20 \\
\mathrm{C}: 45 / 15\end{array}$ & NR \\
\hline Liu et al. [9] & 2002 & $56 / 44$ & $35-67$ & $78 / 2$ & $3-25$ \\
\hline Chen et al. [10] & 2003 & $43 / 42$ & $36-71,54.72$ & NR & NR \\
\hline Cai [11] & 2004 & $66 / 122$ & $\begin{array}{l}\text { T: } 54.6 \\
\text { C: } 58.7\end{array}$ & $\begin{array}{c}\text { T: } 59 / 7 \\
\text { C: } 107 / 15\end{array}$ & NR \\
\hline Wu et al. [12] & 2006 & $37 / 31$ & 35-65 & $51 / 17$ & $>2$ \\
\hline Yi and $\mathrm{Li}[13]$ & 2007 & $45 / 43$ & $\begin{array}{l}\text { T: } 20-55,37 \\
\text { C: } 18-51,33\end{array}$ & $\begin{array}{l}\mathrm{T}: 25 / 20 \\
\mathrm{C}: 25 / 18\end{array}$ & $\begin{array}{l}\mathrm{T}: 5-13 \\
\mathrm{C}: 5-10\end{array}$ \\
\hline Pen and Wang [14] & 2010 & $52 / 44$ & $\begin{array}{l}\text { T: } 28-69,36.8 \\
\text { C: } 24-67,35.6\end{array}$ & $\begin{array}{l}\text { T: } 38 / 14 \\
\text { C: } 28 / 16\end{array}$ & NR \\
\hline Liu et al. [15] & 2010 & $32 / 31$ & $25-62,39.7$ & $\begin{array}{l}\text { T: } 26 / 6 \\
\text { C: } 23 / 8\end{array}$ & NR \\
\hline
\end{tabular}

T: trials group, C: control group. NR: no report.

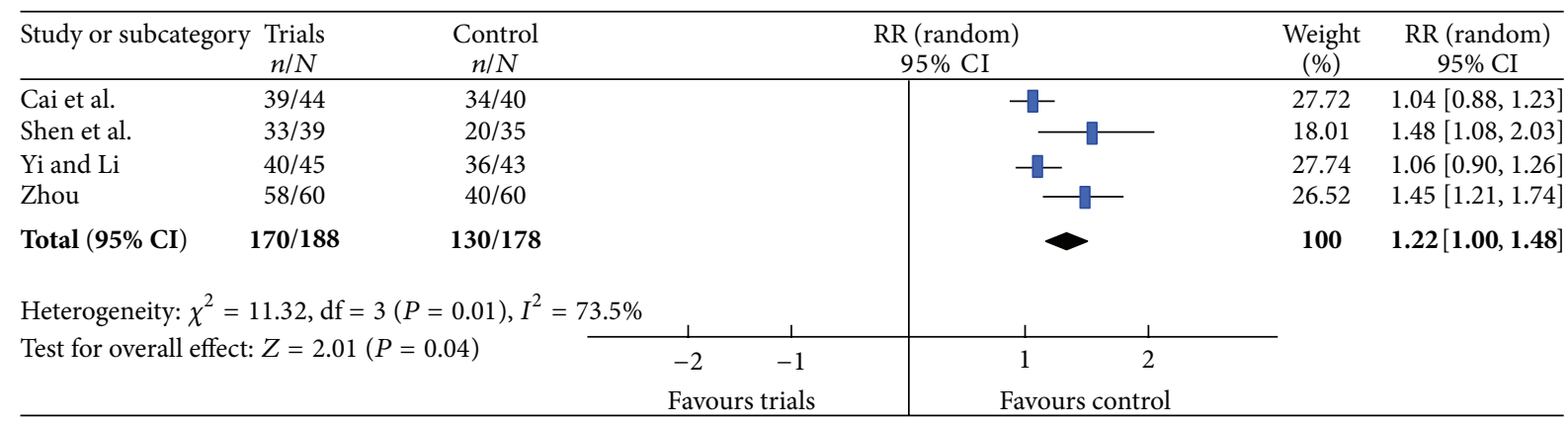

FIGURE 2: Meta-analysis of assessing the total efficacy rate.

vein in the trials group significantly decreased, while the flow velocity of portal vein blood significantly increased $(P<$ .05). Liu et al [15] reported that no difference in the HBeAg seroconversion rate was found between the trials group and the control group $(P>.05)$, while the copies of HBV-DNA in the trials group were significantly lower than those in the control group $(P<.05)$.

3.7. Recurrence Rate. Cai et al. [7] reported that, when 3 months after treatment, 2 cases recurred in the trials group (38 cases received followup); while in the control group (20 cases received followup), 6 cases recurred. There was statistical difference $(P<.05)$ between the two groups.

3.8. Adverse Effect. Amongst 11 literatures included in this study, only one [12] reported side effects and clarified that no side effects in clinical trials were observed.
3.9. Publication Bias. According to the data involving TBIL, the funnel plot was drawn using WMD as abscissa and SE (WMD) for the vertical axis, respectively. The plot was asymmetric (Figure 6), suggesting that the publication biases may occur in this study.

\section{Discussion}

Liver fibrosis results from chronic damage to the liver in conjunction with the accumulation of ECM proteins including collagen, which is a characteristic of most types of chronic liver diseases [16]. In contrast with the traditional view that cirrhosis is an irreversible disease, recent evidence indicates that even advanced fibrosis is reversible $[17,18]$. However, up to now, there has been no standard Western medicine treatment for liver fibrosis. In traditional Chinese medicine (TCM), some Chinese herbs such as Radix Salviae Miltiorrhizae and Radix Astragali Mongolici were commonly 
TABLE 2: The intervention and the outcome measures of the individual studies included in this study.

\begin{tabular}{|c|c|c|c|c|}
\hline \multirow{2}{*}{ Study ID } & \multicolumn{2}{|c|}{ The regimens of intervention } & \multirow{2}{*}{ Time } & \multirow{2}{*}{ Assessment of outcomes } \\
\hline & Trials group & Control group & & \\
\hline Shen et al., 1999 [5] & $\begin{array}{l}\text { Compound Danshen injection } \\
(16 \mathrm{~mL}) \text { and Huangqi injection } \\
(20 \mathrm{~mL}) \text { were, respectively, added } \\
\text { into } 5 \%-10 \% \text { glucose solution } \\
(250 \mathrm{~mL}) \text {, intravenously, qd. }\end{array}$ & $\begin{array}{l}\text { Aspartic potassium, } \\
\text { vitamin C, and B6 were } \\
\text { added into 5\% glucose, } \\
\text { intravenously. }\end{array}$ & $28 \mathrm{~d}$ & $\begin{array}{l}\text { Total efficacy rate; serum } \\
\text { indices of liver fibrosis } \\
\text { and liver function. }\end{array}$ \\
\hline Chen et al., 2000 [6] & $\begin{array}{l}\text { Compound Danshen injection } \\
(20 \mathrm{~mL}) \text { and Huangqi injection } \\
(20 \mathrm{~mL}) \text { were, respectively, added } \\
\text { into } 5 \% \text { glucose solution, } \\
\text { intravenously, qd. }\end{array}$ & $\begin{array}{l}\text { Used hepatoprotective } \\
\text { treatment (mainly energy } \\
\text { mixture and vitamins), } \\
\text { qd. }\end{array}$ & $90 \mathrm{~d}$ & $\begin{array}{l}\text { Serum indices of liver } \\
\text { fibrosis. }\end{array}$ \\
\hline Cai et al., 2001 [7] & $\begin{array}{l}\text { Compound Danshen injection } \\
(20 \mathrm{~mL}) \text { and Huangqi injection } \\
(20 \mathrm{~mL}) \text { were, respectively, added }\end{array}$ & Used silymarin orally, tid. & $90 \mathrm{~d}$ & $\begin{array}{l}\text { Total efficacy rate; serum } \\
\text { indices of liver function. }\end{array}$ \\
\hline
\end{tabular}
into $5 \%$ glucose solution,

intravenously, qd.

Compound Danshen injection

$(20 \mathrm{~mL})$ and Huangqi injection

Zhou, $2002[8] \quad(10 \mathrm{~mL})$ were together added into

$250 \mathrm{~mL}$ of $5 \%$ glucose solution, intravenously, qd.

Danshen injection $(30 \mathrm{~mL})$ and Huangqi injection $(20 \mathrm{~mL})$ were,

Liu et al., 2002 [9] respectively, added into $250 \mathrm{~mL}$ of $5 \%$ glucose solution, intravenously, qd.

Danshen injection $(30 \mathrm{~mL})$ and Huangqi injection $(16 \mathrm{~mL})$ were,

Chen et al., 2003 [10] respectively, added into $250 \mathrm{~mL}$ of $5 \%$ glucose solution, intravenously, qd.

Compound Danshen injection $(30 \mathrm{~mL})$ and Huangqi injection

Cai, 2004 [11] $(10 \mathrm{~mL})$ were, respectively, added into $250 \mathrm{~mL}$ of $5 \%$ glucose solution, intravenously, qd.

Danshen injection $(25 \mathrm{~mL})$ and Huangqi injection $(25 \mathrm{~mL})$ were,

Wu et al., 2006 [12] respectively, added into $250 \mathrm{~mL}$ of $5 \%$ glucose solution, intravenously, qd.

Danshen injection $(20 \mathrm{~mL})$ and Huangqi injection $(20 \mathrm{~mL})$ were, respectively, added into $250 \mathrm{~mL}$ of $5 \%$ glucose solution, intravenously, qd.

Danshen injection $(30 \mathrm{~mL})$ and Huangqi injection $(30 \mathrm{~mL})$ were,

Pen and Wang, 2010 [14] respectively, added into $250 \mathrm{~mL}$ of $5 \%$ glucose solution, intravenously, qd.

Danshen injection $(30 \mathrm{~mL})$ and Huangqi injection $(30 \mathrm{~mL})$ were, Liu et al., 2010 [15] respectively, added into $250 \mathrm{~mL}$ of $10 \%$ glucose solution, intravenously, qd.
Vitamin C, vitamin B6, inosine, and potassium chloride injection were added into $5 \%$ glucose solution intravenously, qd.

Added ganlixin into the $5 \%$ glucose, intravenously.

$90 \mathrm{~d}$

$30 \mathrm{~mL}$ of ganlixin

injection, intravenously, qd.

Energy mixture, branched-chain amino acids, albumin, and vitamin were used.

Routine western medicine treatment.

$84 \mathrm{~d}$

Used silymarin orally, 3 times a day.

$90 \mathrm{~d}$

Hepatoprotective therapy using nosine and vitamins.

Hepatoprotective and supportive therapy and lamivudine $(100 \mathrm{mg}$ ) orally, qd. indices of liver function.

Total efficacy rate; serum indices of liver function.

Serum indices of liver function.

Serum indices of liver fibrosis and liver function.

Serum indices of liver fibrosis and liver function.

Serum indices of liver function; liver hemodynamics.

Total efficacy rate; serum indices of liver function.

Serum indices of liver fibrosis and liver function.

Serum indices of liver fibrosis and liver function; $\mathrm{HBeAg}$; HBV-DNA.

The regimens in the trials group included the drugs used in the control group. Compound Danshen injection: mainly contains Danshen and an added constituent (Lignum Dalbergiae Odoriferae). 


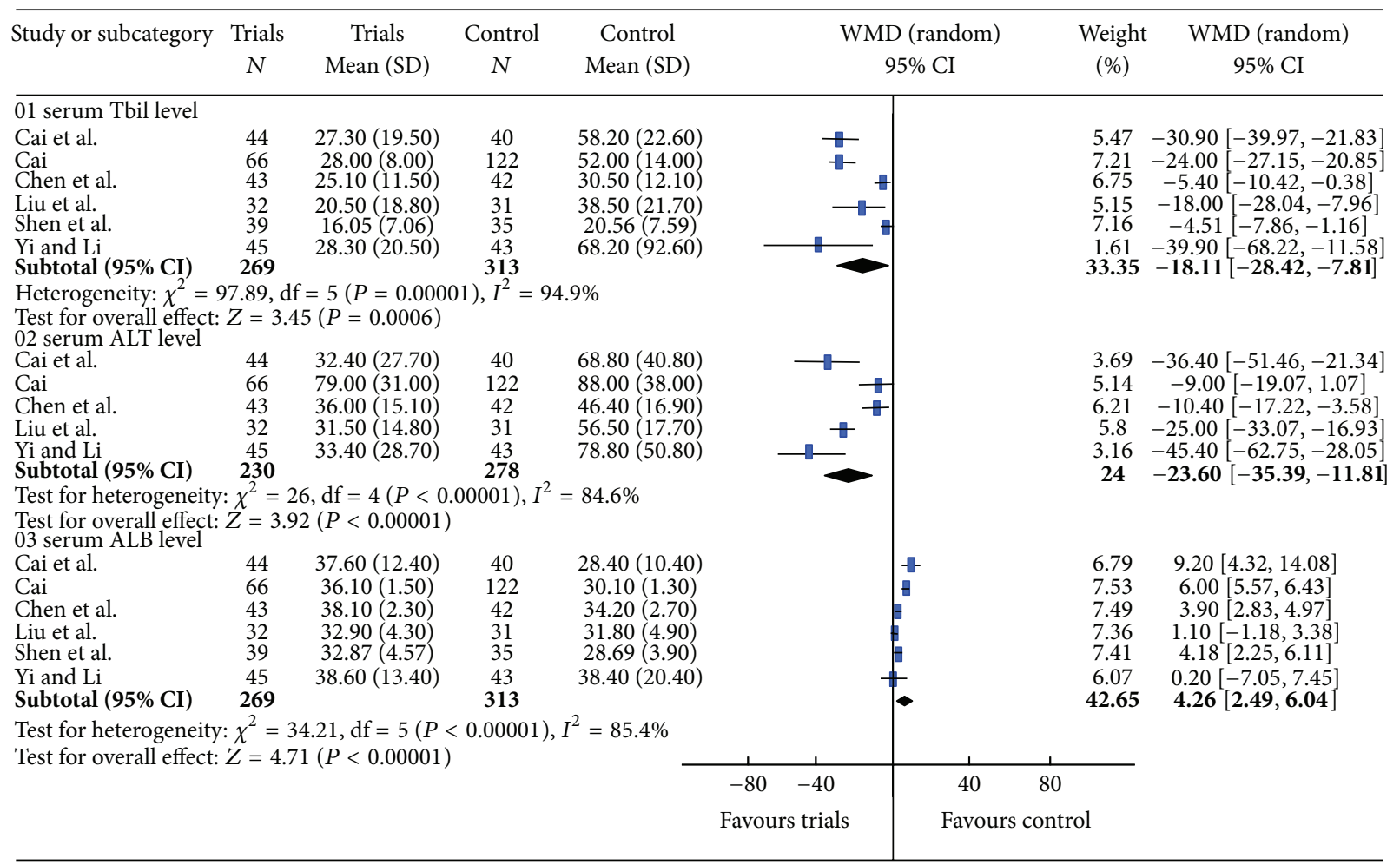

FIgURE 3: Meta-analysis of the serum indices of liver function (TBIL, ALT and ALB).

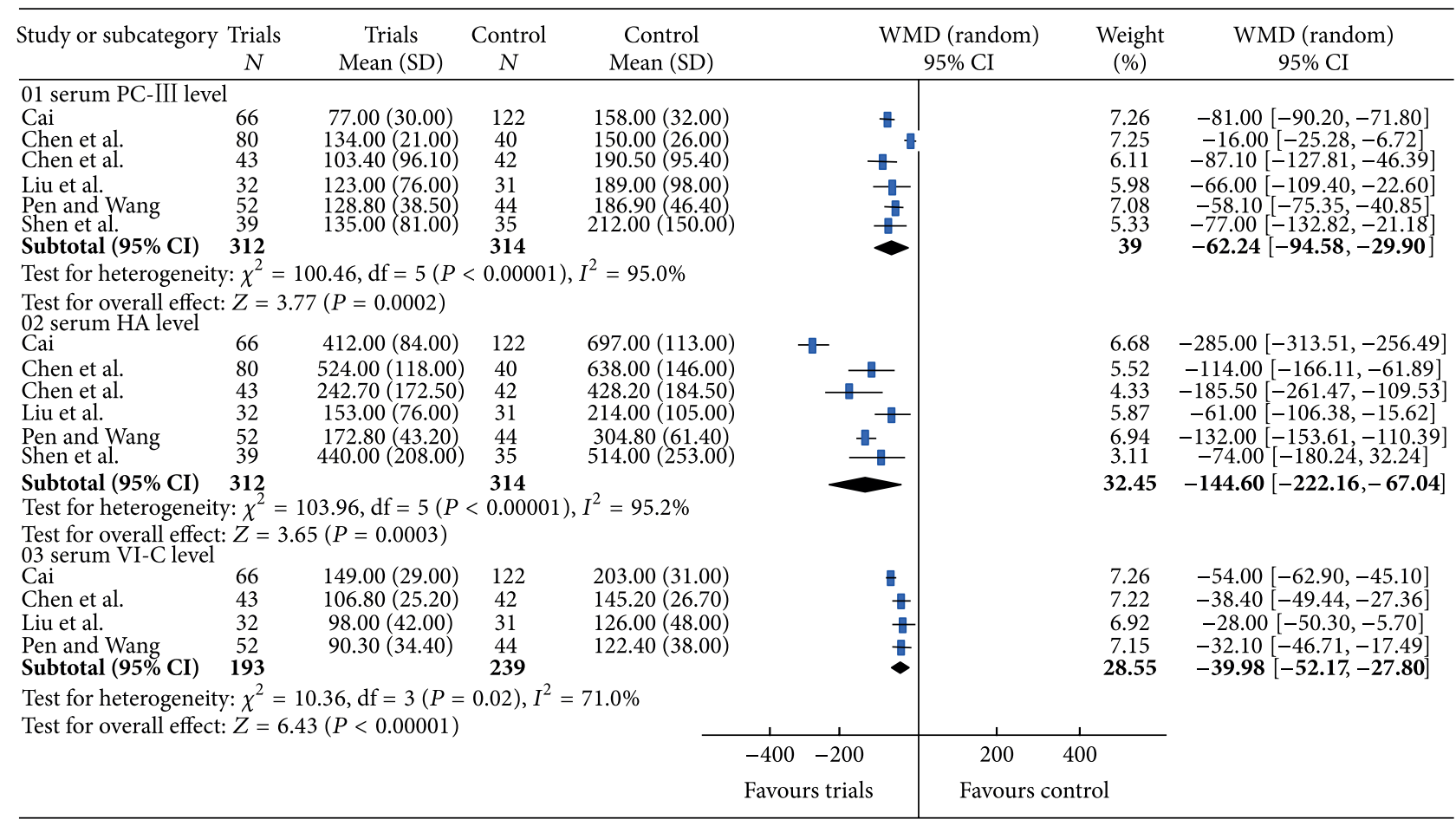

FIGURE 4: Meta-analysis of the serum indices of liver fibrosis (PCIII, HA and CIV). 
TABLE 3: Quality of reports of 11 clinical trials using the Jadad assessment scale.

\begin{tabular}{|c|c|c|c|c|c|}
\hline & Randomization & Double blinding & Withdrawals/dropouts & Allocation concealment & Scores \\
\hline Curative effect assessment & Yes & No & No & No & 1 \\
\hline Serum indices of liver function & Yes & Yes & No & No & 2 \\
\hline Serum indices of liver fibrosis & Yes & Yes & No & No & 2 \\
\hline
\end{tabular}

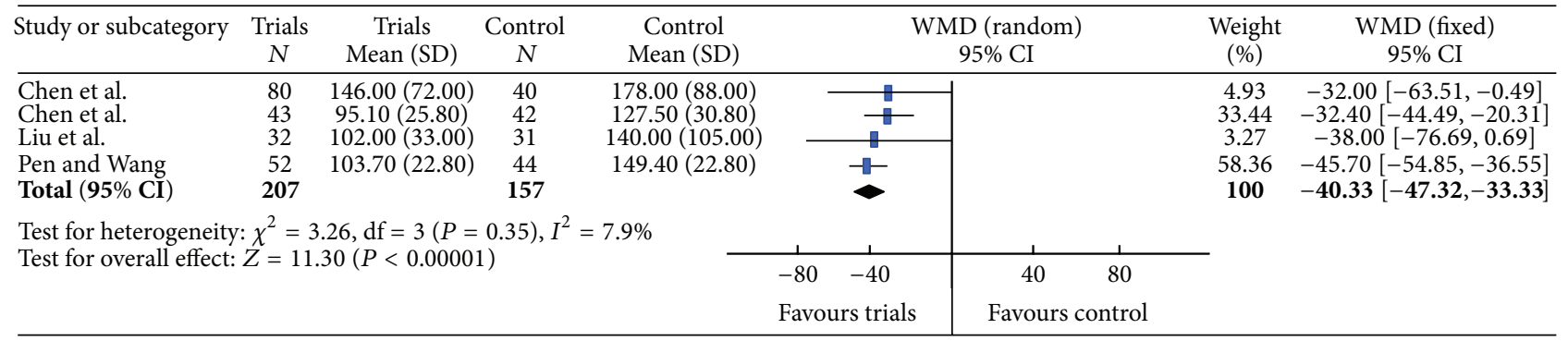

FIGURE 5: Meta-analysis of the indices of liver fibrosis (LN).

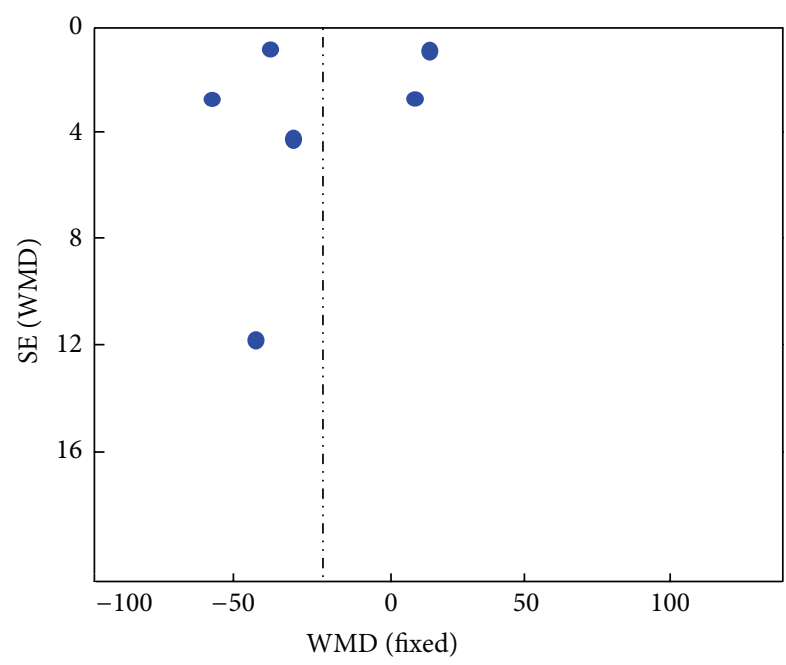

FIgURE 6: The funnel plot based on the data of TBIL.

used in cases of liver fibrosis and hepatitis. In recent years, the extract of their roots using ethanol was prepared as Danshen injection and Huangqi injection, respectively, which were often prescribed as the treatment for liver fibrosis. However, in China, Danshen injection was commonly used for coronary heart disease [19], myocarditis [20], stroke disease [21], myocardial infarction [22], hepatitis [23], epidemic hemorrhagic fever [24], acute disseminated intravascular coagulation syndrome so on [25]. Huangqi injection has been widely used in the treatment for chronic hepatitis [26], viral myocarditis [27], chronic heart failure [28], aplastic anemia [29], chronic nephritis [30], renal damage [31], nephrotic syndrome [32, 33], and diabetic nephropathy [34].

Radix Salviae Miltiorrhizae contains tanshinones, salvianolic acids, rosmarinic acid, lithospermic acid, protocatechualdehyde, caffeic acid, isoferulic acid, and so forth. It has been demonstrated that the ingredients can inhibit the proliferation and activation of stellate cells, and promote the repair and regeneration of liver cells, improve microcirculation in the liver, and increase hepatic parenchymal blood supply [35, 36]. Modern pharmacological studies have shown that, Radix Astragali Mongolici contains a series of cycloartane triterpene glycosides denoted astragalosides I-VII (saponins), which can inhibit the synthesis and the deposition of collagen, and promote the protein synthesis and the recovery of the cell function in liver [37-39].

In this study, Danshen injection and Huangqi injection combined with routine therapeutic regiment (the control) were used for the clinical treatment of cirrhosis. The treatment protocols were as follows: Danshen injection (10$30 \mathrm{~mL}$ ) and Huangqi injection $(10-30 \mathrm{~mL})$ were respectively or together added into $250-500 \mathrm{~mL}$ of $5 \%-10 \%$ glucose solution for intravenous drip, qd, and the course of treatment ranges from $28 \mathrm{~d}$ to $90 \mathrm{~d}$. Meta-analysis showed that the therapeutic effect in the trials group (using Danshen injection and Huangqi injection) was better than that in the control group (routine treatment). Serum ALT, ALB, and TBIL are the main markers of evaluating liver function, and the metaanalysis implied that using Danshen injection and Huangqi injection can decrease the serum levels of ALT, ALB, and TBIL, suggesting that the injections improve recovery of liver function. HA, LN, VI-C, and PC-III are the serum markers of the therapeutic evaluation of liver fibrosis; meta-analysis results showed that; the serum levels of HA, LN, VI-C, and PC-III in the trials group were lower compared with those in the control group. Thus, the treatment was believed to relieve liver fibrosis. In addition, individual study [12] showed that, after 4 weeks of treatment using Danshen injection and Huangqi injection, portal vein diameter, the inner diameter, and blood flow velocity of the splenic vein decreased, while the flow velocity of portal vein blood increased, indicating that the liver thermodynamics were bettered after the treatment using the injections. In addition, Liu et al. [15] reported 
that the copies of HBV-DNA had significantly reduced after the treatment.

Based on the above results, we believed that using Danshen injection and Huangqi injection combined with the routine treatment may improve liver function recovery and reverse liver fibrosis. But, it must be noted that this metaanalysis had some limitations. Firstly, the quality of the methodological design of individual studies was not high. Secondly, the asymmetric funnel plot implied that publication biases may occur. Thirdly, the diversity of treatment dose and the small sample number and the lack of longterm follow-ups degraded the validity of the evidence of the clinical trials. In addition, the safety of the trials was reported insufficiently, and there only had one literature that mentioned side effect and none else clarified the safety observation. However, the previous study [40] reported that, using Huangqi injection in treating chronic hepatitis B patients showed flustered symptoms, and the symptoms disappeared after slowing drip rate. In addition, another study reported that the rarely moderate anaphylaxis in intravenous dripping of Danshen injection may occur [41].

\section{Conclusions}

Danshen injection combined with Huangqi injection may promote the curative efficacy of liver cirrhosis, which is a promising novel treatment approach. Considering that this systematic review had the limitations in some ways, rigorously designed multicenter, double-blind, randomized, and large-scale controlled trials are required.

\section{Conflict of Interests}

The authors declare that they have no conflict of interests.

\section{Authors' Contribution}

Changtai Zhu, Hao Cao, and Xifa Zhou contributed equally to this work.

\section{Acknowledgments}

This work was supported by the National Natural Science Foundation of China (no. 81071799), the Science and Technology Bureau of Changzhou Municipality (nos. CJ2012202 and CE20125026), and the Preventive Medicine Project of the Provincial Public Health Bureau of Jiangsu (No. Y2012095), and the Special Funding of Innovation and Transformation of Science and Technology of Jiangsu Province (no. BL2012046).

\section{References}

[1] W. J. Dai and H. C. Jiang, "Advances in gene therapy of liver cirrhosis: a review," World Journal of Gastroenterology, vol. 7, no. 1, pp. 1-8, 2001.

[2] R. Bataller and D. A. Brenner, "Liver fibrosis," Journal of Clinical Investigation, vol. 115, no. 2, pp. 209-218, 2005.
[3] J. Liu and D. Fan, "Hepatitis B in China," The Lancet, vol. 369, no. 9573, pp. 1582-1583, 2007.

[4] B. Custer, S. D. Sullivan, T. K. Hazlet, U. Iloeje, D. L. Veenstra, and K. V. Kowdley, "Global epidemiology of hepatitis B virus," Journal of Clinical Gastroenterology, vol. 38, no. 10, supplement 3, pp. S158-S168, 2004.

[5] Q. Shen, Q. W. Sun, Z. Zhang et al., “The treatment for 39 cases of cirrhosis with compound Danshen injection and Huangqi injection," Shang Hai Yi Yao, vol. 20, no. 4, pp. 26-28, 1999.

[6] X. Chen, X. Li, and M. Liu, "Compound Danshen Huangqi injection treatment for 80 cases of liver fibrosis," Zhong Xi Yi Jie He Gan Bing Za Zhi, vol. 10, no. 3, p. 39, 2000.

[7] Y. J. Cai, Y. P. Gao, Q. Q. Zhang et al., "Compound Huangqi Danshen injection in the treatment for 44 cases of liver cirrhosis caused by hepatitis chronic hepatitis," Zhongguo Zhong Xi Yi Jie He Za Zhi, vol. 21, no. 7, pp. 548-549, 2001.

[8] C. X. Zhou, "The treatment for 120 cases cirrhosis caused by hepatitis compound with Danshen combined with Huangqi," Xian Dai Zhong Xi Yi Jie He Za Zhi, vol. 11, no. 21, pp. 2111-2112, 2002.

[9] Y. Q. Liu, W. Han, Z. J. Wang, L. X. Li, and S. Q. Gao, “The efficacy of Huangqi combined with Danshen injection in the treatment for liver cirrhosis," Ji Lin Zhong Yi Yao, vol. 22, no. 5, p. 15, 2002.

[10] J. Chen, Z. Chen, X. J. Huang et al., “The effect on serum markers reflecting the liver function and liver fibrosis in the treatment for liver cirrhosis with Danshen, Huangqi injection," An Hui Zhong Yi Xue Yuan Xue Bao, vol. 22, no. 5, pp. 32-34, 2003.

[11] Y. Cai, "The treatment with Danshen combined with Huangqi injection for 66 cases of liver cirrhosis," Shan Xi Yi Yao Za Zhi, vol. 33, no. 4, pp. 342-343, 2004.

[12] A. M. Wu, L. M. Zhang, H. Tang et al., "Liver hemodynamics of cirrhosis resulted from hepatitis B liver of the treatment with Danshen and Huangqi injection intravenous infusion," Shi Jie Gan Ran Za Zhi, vol. 6, no. 1, pp. 40-42, 2006.

[13] S. H. Yi and Y. F. Li, "The treatment with Huangqi injection and Danshen injection for 45 cases of cirrhosis caused by chronic hepatitis," Zhe Jiang Zhong Yi Za Zhi, vol. 42, no. 4, p. 243, 2007.

[14] A. Pen and Z. Y. Wang, "Clinical observation on Huangqi injection and Danshen injection in the treatment for 52 cases of patients," Zhongguo Yi Yuan Yao Xue Za Zhi, vol. 30, no. 13, pp. 1131-1133, 2010.

[15] S. J. Liu, W. J. Lin, D. J. Tu et al., "The treatment for decompensated cirrhosis due to chronic hepatitis B liver with Danshen injection, Huangqi injection combined with lamivudine," Zhongguo Zhong Yi Ji Zhen, vol. 19, no. 6, pp. 934-935, 2010.

[16] F. Cheng, Y. Li, L. Feng, and S. Li, "Hepatic stellate cell activation and hepatic fibrosis induced by ischemia/reperfusion injury," Transplantation Proceedings, vol. 40, no. 7, pp. 2167-2170, 2008.

[17] M. J. P. Arthur, "Reversibility of liver fibrosis and cirrhosis following treatment for hepatitis C," Gastroenterology, vol. 122, no. 5, pp. 1525-1528, 2002.

[18] V. Soriano, P. Labarga, A. Ruiz-Sancho, J. Garcia-Samaniego, and P. Barreiro, "Regression of liver fibrosis in hepatitis C virus/HIV-co-infected patients after treatment with pegylated interferon plus ribavirin," AIDS, vol. 20, no. 17, pp. 2225-2227, 2006.

[19] L. Y. Pei, M. C. Zheng, and H. L. Liang, "Meta-analysis of curative effect of Danshen injection compared with puerarin on coronary heart disease," He Nan Zhong Yi, vol. 28, no. 7, pp. 9192, 2008. 
[20] X. Q. Wang and Y. Zhang, "Shengmai injection combined with Compound Danshen injection in the treatment for 64 cases of children with acute viral myocarditis," Zhongguo Zhong Yi Ji Zhen, vol. 20, no. 7, pp. 1184-1185, 2011.

[21] X. Yang and C. M. Du, "The Medofenoxate and Huangqi injection in the treatment for 68 cases of patients with cerebral vascular accident," Si Chuan Zhong Yi, vol. 20, no. 1, p. 43, 2002.

[22] H. W. Lin and H. Q. Peng, "Effect of compound Danshen injection on t-PA, PAI and GMP-140 in the patients with acute myocardial infarction," Zhongguo Zhong Yi Ji Zhen, vol. 14, no. 7, pp. 615-616, 2005.

[23] L. J. Ye, "Efficacy observation of Danshen injection combined with Shenmai injection in treating chronic hepatitis B," Hai Xia Yao Xue, vol. 24, no. 6, pp. 181-182, 2012.

[24] Z. H. Xu, "The application of compound Danshen injection in epidemic hemorrhagic fever," Xin Zhong Yi, vol. 15, no. 3, pp. 38-39, 1995.

[25] S. L. Yang, D. Li, and J. Y. Yan, "A large dose of compound Danshen injection in the treatment of 24 cases of patients with acute leukemia complicated with disseminated intravascular coagulation," Zhongguo Zhong Xi Yi Jie He Za Zhi, vol. 20, no. 3, pp. 226-227, 2000.

[26] Y. L. Bai, "Therapeutic effect of danshen injection on chronic active hepatitis," Zhong Xi Yi Jie He Za Zhi, vol. 4, no. 2, pp. 8687, 1984.

[27] M. He, M. L. Luo, L. J. Chen et al., "System review of Huangqi injection in the treatment for viral myocarditis," Zhongguo Yao Fang, vol. 21, no. 48, pp. 4570-4575, 2010.

[28] S. Fu, J. Zhang, F. Menniti-Ippolito et al., "Huangqi injection (a traditional chinese patent medicine) for chronic heart failure: a systematic review," PLoS One, vol. 6, no. 5, Article ID e19604, 2011.

[29] R. Chao, Y. M. Xi, and R. R. Jin, "The evaluation of clinical efficacy and safety of aplastic anemia in treatment of Huangqi injection," Zhongguo Xun Zheng Yi Xue, vol. 11, no. 10, pp. 12051209, 2011.

[30] S. L. Wang and C. L. Guo, "Clinical efficacy analysis of huangqi injection in the treatment 15 cases of patients with chronic nephritis," Ji Lin Yi Xue, vol. 33, no. 30, p. 6549, 2012.

[31] T. Sun, H. Xu, and F. Xu, "Astragalus injection for hypertensive renal damage: a systematic review," Evidence-Based Complementary and Alternative Medicine, vol. 2012, Article ID 929025, 6 pages, 2012.

[32] Y. W. Bai, "Clinical observation of Huangqi injection in treatment of primary nephrotic syndrome," An Hui Yi Xue, vol. 25, no. 4, pp. 299-300, 2004.

[33] J. R. Lin and C. Deng, "Clinical study of Huangqi injection in the treatment of children with nephrotic syndrome," Shi Zhen Guo Yi Guo Yao, vol. 18, no. 1, pp. 169-170, 2007.

[34] M. Li, W. Wang, J. Xue, Y. Gu, and S. Lin, "Meta-analysis of the clinical value of Astragalus membranaceus in diabetic nephropathy," Journal of Ethnopharmacology, vol. 133, no. 2, pp. 412-419, 2011.

[35] J. W. Xu and C. M. Fu, "Advances in the pharmacologics of Salvia miltiorrhiza," Yi Xue Zong Shu, vol. 12, no. 23, pp. 1467-1469, 2006.

[36] Y. Gu, W. Gu, and S. L. Yu, "Research progress on pharmacological action of Salvia miltiorrhiza," Ren Shen Yan Jiu, vol. 19, no. 1, pp. 25-27, 2007.

[37] Y. Z. Liu, D. L. Ma, and J. Gao, "The protective effects of Huangqi injection on immunological liver injury in mice," Tian Jin Yi Ke Da Xue Xue Bao, vol. 10, no. 2, pp. 190-191, 2004.
[38] G.-H. Chen and W.-F. Huang, "Progress in pharmacological effects of compositions of Astragalus membranaceus," Zhongguo Xin Yao Za Zhi, vol. 17, no. 17, pp. 1482-1485, 2008.

[39] Y. J. Wang, Q. Z. Quan, and S. Ziqin, "Research on the immune effect of Astragalus membranaceus on ICAM-1 expression in liver fibrosis," Zhongguo Lin Chuang Yao Li Xue Yu Zhi, vol. 5, no. 1, pp. 49-51, 2000.

[40] Z. X. Sun, A. P. Wang, and X. Y. Dong, "Huangqi injection in the treatment of chronic hepatitis B: analysis of 60 cases," Shan Dong Yi Xue, vol. 41, no. 2, p. 38, 2001.

[41] M. L. Wen and Y. Han, "Observation on the clinical safety of Danshen injection," Zhongguo Fang Ji Xue Za Zhi, vol. 18, no. 22, pp. 322-324, 2012. 


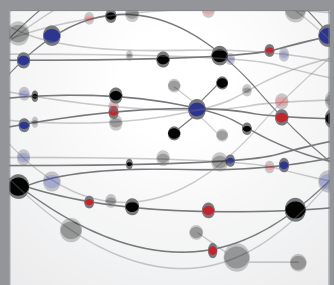

The Scientific World Journal
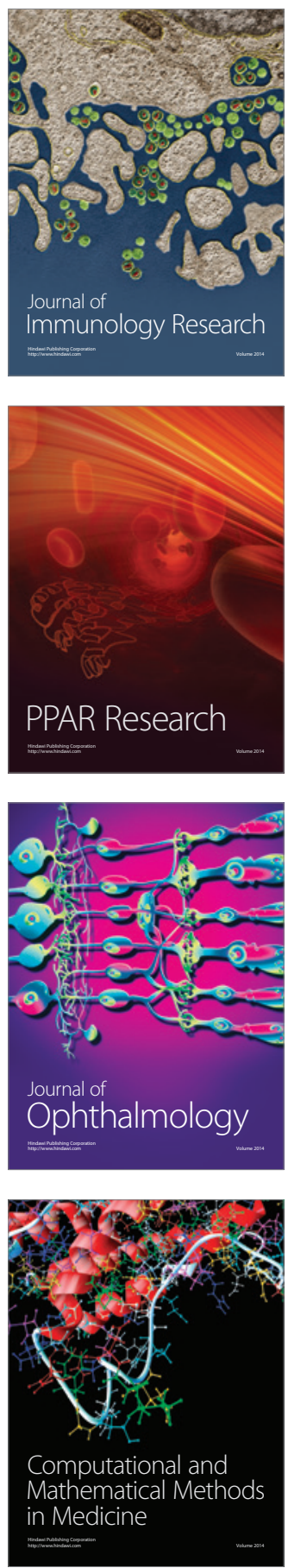

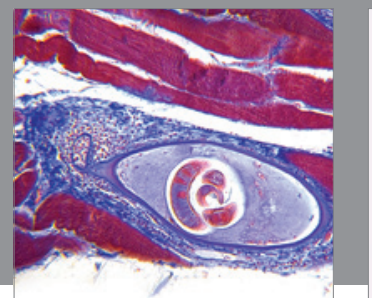

Gastroenterology

Research and Practice
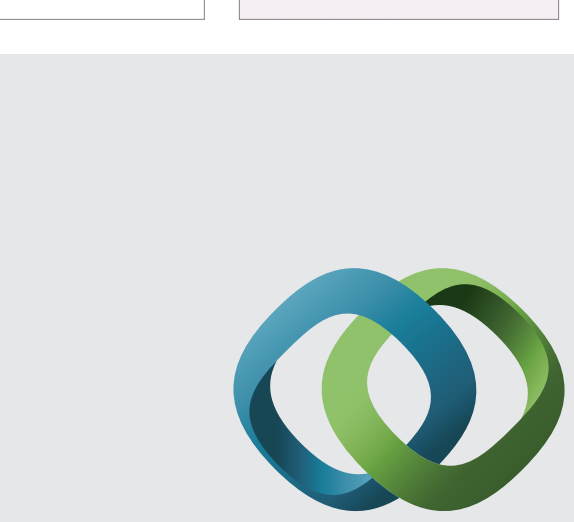

\section{Hindawi}

Submit your manuscripts at

http://www.hindawi.com
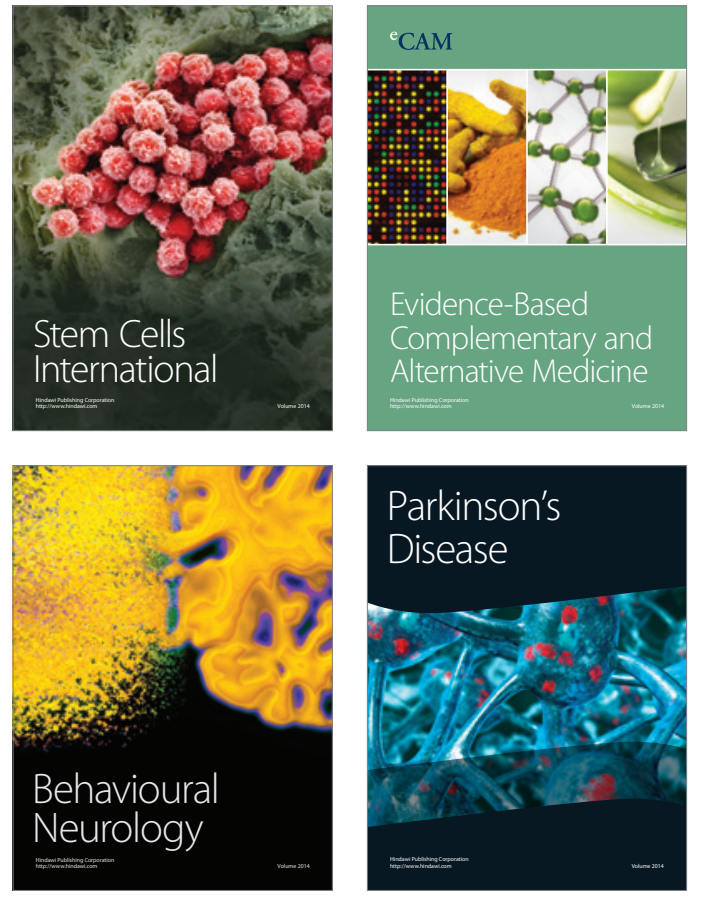
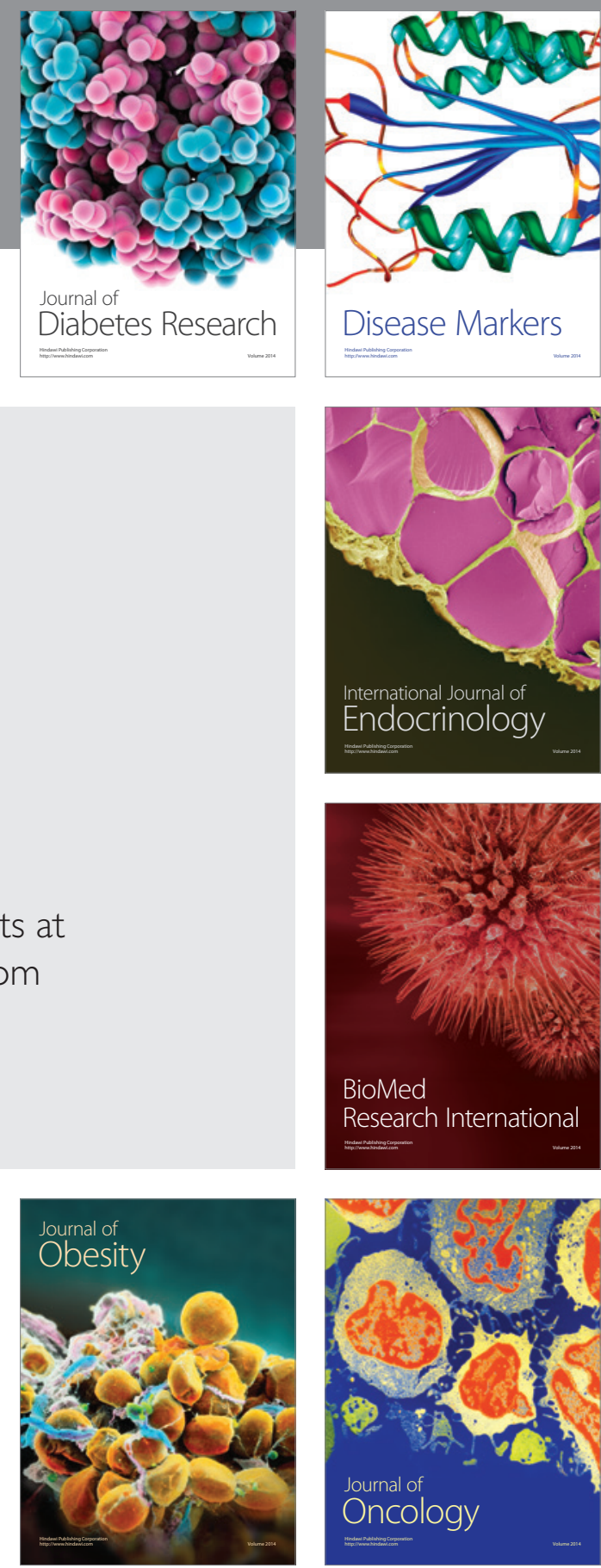

Disease Markers
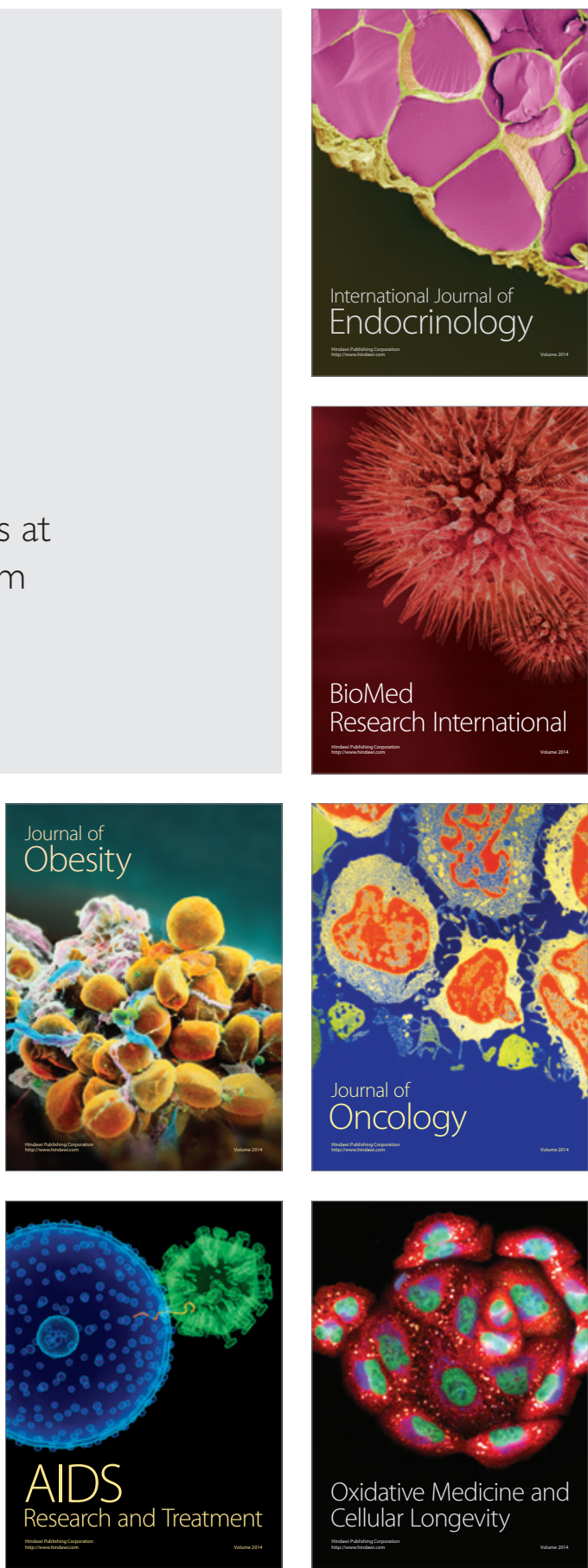